\title{
Results of management of spinal tuberculosis according to middle path regime and short course chemotheraphy
}

\author{
Jairam D. Jagiasi ${ }^{1}$, Mihir R. Patel $^{2} *$
} Department of Orthopaedics, ${ }^{1}$ B. Y. L. Nair Charitable Hospital and T. N. Medical College, ${ }^{2}$ H. B. T. Medical College
and Dr R. N. Cooper Hospital, Juhu, Mumbai, Maharashtra, India

Received: 16 July 2017

Revised: 01 August 2017

Accepted: 03 August 2017

\author{
*Correspondence: \\ Dr. Mihir R. Patel, \\ E-mail: mrpatel1981@gmail.com
}

Copyright: (c) the author(s), publisher and licensee Medip Academy. This is an open-access article distributed under the terms of the Creative Commons Attribution Non-Commercial License, which permits unrestricted non-commercial use, distribution, and reproduction in any medium, provided the original work is properly cited.

\begin{abstract}
Background: Vertebral tuberculosis is the most common form of skeletal tuberculosis and it contributes about 50 percent of all cases of skeletal tuberculosis. The purpose of this study is to delineate the importance of middle path regime and short course chemotheraphy in the management of spine tuberculosis.

Methods: A prospective study was conducted which included 44 patients diagnosed as tuberculous spondylitis. Management was started as per middle path regime and drugs were given as per the revised national tuberculosis control program (RNTCP) regime category I under directly observed treatment, short-course (DOTS) therapy. Specimen was collected and studied for smear, culture, antibiotic sensitivity and histopathology. ATT was stopped at the end of 6 months as per DOTS schedule and MRI was done at the end of therapy.

Results: Dorsal spine lesions were found in 33 patients. 8 patients were smear positive and 38 patients were culture positive of whom 26 patients were sensitive to first line ATT drugs. 12 patients had MDR tuberculosis and were sensitive to $2^{\text {nd }}$ line ATT drugs. 6 patients having negative culture were diagnosed from histopathology. All patients on $1^{\text {st }}$ line ATT had satisfactory result. Only 8 patients with MDR tuberculosis recovered neurologically.

Conclusions: It is mandatory to do obtain tissue biopsy and examine culture, sensitivity and histopathology. Recently there is an increasing tendency in multidrug resistance cases and total drug resistant cases. By doing culture and sensitivity of the specimen we can find out the number of MDR cases and initiate proper drug regimen to avoid further complication and mortality.
\end{abstract}

Keywords: Tuberculosis, Spine, Anti tubercular therapy, Middle path regime

\section{INTRODUCTION}

The world at large has nearly 30 million people suffering from tuberculosis. ${ }^{1}$ Due to marked improvement in the socio-economic status of affluent countries and the availability of extremely effective antitubercular drugs up to early 1980s, there was great hope for complete elimination of the disease. Unfortunately the optimism was short-lived because of the impact of acquired immunodeficiency syndrome (AIDS) pandemic. Tuberculosis has again become epidemic in many parts of the world. After 1985, many affluent countries were recording increase in the number of patients by 10 to 20 percent annually. According to current estimates of WHO tuberculosis now kills 3 million people a year worldwide. It is estimated that India alone has got one-fifth of the total world population of tuberculous patients.

Like other skeletal structures, vertebral column may lodge any infectious process. Most common chronic vertebral infection, however, is tuberculosis. Vertebral tuberculosis is the most common form of skeletal 
tuberculosis and it contributes about 50 percent of all cases of skeletal tuberculosis. ${ }^{1}$ The major areas of predilection are in following order-spine, hip, knee, foot, elbow, hand, shoulder, bursal sheaths and others.

The purpose of this study is to delineate the importance of middle path regime and short course chemotheraphy in the management of spine tuberculosis. Patients were followed up at monthly interval for 6 months and then every 2 months for the next 1 year. Patients were clinically assessed based on symptoms and signs.

\section{METHODS}

This was a prospective study conducted in the department of orthopaedics at a tertiary level hospital in the time period of January-2012 to December-2012. Patients diagnosed as tuberculous spondylitis in the outpatient department or admitted patients in the orthopaedic ward having the following criteria were included in the study:

1. Microbiologically and histopathological diagnosed cases of tuberculosis spondylitis and

2. Patients willing for follow up till the completion of study.

Exclusion criteria included patients not willing to be part of the study. Forty four patients were included in the study.

Table 1: Paediatric doses in different weight bands as recommended by RNTCP.

\begin{tabular}{|lllll|}
\hline Weight band (in kg) & Rifampicin (mg) & Isoniazid (mg) & Pyrazinamide (mg) & Ethambutol (mg) \\
\hline $\mathbf{6 - 1 0}$ & 75 & 75 & 250 & 200 \\
\hline $\mathbf{1 1 - 1 7}$ & 150 & 150 & 500 & 400 \\
\hline $\mathbf{1 8}-\mathbf{2 5}$ & 225 & 225 & 750 & 600 \\
\hline $\mathbf{2 6}-\mathbf{3 0}$ & 300 & 300 & 1000 & 800 \\
\hline
\end{tabular}

Table 2: Frankel grading for spinal cord function.

\begin{tabular}{|ll|}
\hline Grade & Spinal cord function \\
\hline A & Complete paralysis \\
\hline B & Sensory function only below the affected level \\
\hline C & Incomplete motor function below affected level \\
\hline D & Fair to good motor function below affected level \\
\hline
\end{tabular}

Table 3: Drugs used as $2^{\text {nd }}$ line ATT.

\begin{tabular}{|ll|}
\hline Name of the drug & Dosage \\
\hline Amikacin & $15 \mathrm{mg} / \mathrm{kg} / \mathrm{day}$ \\
\hline Aminosalicyclic acid & $8-12 \mathrm{mg} / \mathrm{kg} / \mathrm{day}$ \\
\hline Capreomycin & $15 \mathrm{mg} / \mathrm{kg} / \mathrm{day}$ \\
\hline Ciprofloxacin & $1500 \mathrm{mg} /$ day $($ divided $)$ \\
\hline Clofazimine & $200 \mathrm{mg} /$ day \\
\hline Cycloserine & $500-1000 \mathrm{mg} /$ day $($ divided $)$ \\
\hline Ethionamide & $500-750 \mathrm{mg} /$ day \\
\hline Levofloxacin & $500 \mathrm{mg} /$ day \\
\hline Rifabutin & $300 \mathrm{mg} /$ day \\
\hline
\end{tabular}

The patients were assessed on the basis of their history, clinical symptoms, clinical signs and radiological examination. Management of tuberculosis was started as per middle path regime. ${ }^{2}$ All patients were given high protein diet. Drugs were given as per revised national tuberculosis control program (RNTCP) regime Category I under directly observed treatment, short-course (DOTS) therapy i.e. Isoniazid (H) (600 mg), Rifampicin (R) (450 $\mathrm{mg}$ ), Pyrazinamide (P) (1500 mg), Ethambutol (E) (1200 $\mathrm{mg})$, Streptomycin (S) $(750 \mathrm{mg})$. $^{3}$ Patients who weighed $60 \mathrm{~kg}$ or more receive additional $150 \mathrm{mg}$ of Rifampicin. Patients who were more than 50 years old received only
$500 \mathrm{mg}$ of Streptomycin. Patients who weighed less than $30 \mathrm{~kg}$ received drugs as per body weight (Table 1). All patients were started on HRZE thrice weekly for 2 months and HR for 4 months. If the patient had previous history of tuberculosis, they were given HRZES for 2 months followed by HRZE for 1 month followed by 5 months of HRE.

Patients were followed up at monthly interval for 6 months and then every 2 months for the next 1 year. Patients were clinically assessed based on symptoms and signs. Neurological power was graded as per Frankel 
stage (Table 2). Erythrocyte sedimentation rate (ESR) was done every month and noted. Antero-posterior and lareral radiographs were taken and following details were noted:

- Loss of vertebral height

- Loss of disc space

- Paravertebral abscess

- Soft tissue calcification

- Erosions of vertebral body

Gradual mobilization of the patient was encouraged, in the absence of neurologic deficit, with the help of suitable spinal braces as soon as the patient was comfortable with respect to pain. After 3 to 9 weeks of starting of the treatment the patient was put on back extension exercises 5 to 10 minutes three to four times a day. Spinal brace was continued for 18 months to 2 years after which it was discontinued.

Any abscess was aspirated when near the surface. CT guided biopsy was done if abscess was deeply situated. Drainage was always done whenever decompression surgery was performed. Specimen was sent for smear, culture and antibiotic sensitivity to the microbiology department and for histopathology to the pathology department. Material collected for histopathology was sent in a container containing formalin sufficient enough to submerge the tissue. Material collected for acid fast bacilli (AFB) smear, culture and sensitivity was sent in a sterile test tube containing $2-3 \mathrm{ml}$ of normal saline. Culture was done using the Bactec MGIT 960 TB system to detect Mycobacterium tuberculosis complex (M.tuberculosis, M.bovis, M.africanum, M.microti). As per the sensitivity report, if the patient was sensitive to $1^{\text {st }}$ line Anti Tubercular Therapy (ATT), then same was continued for 6 months. If the patient showed resistance to $1^{\text {st }}$ line ATT then $2^{\text {nd }}$ line ATT (as per the sensitivity report) was given for 18-24 months (Table 3).

Surgery was indicated for a patient in case of the following reasons:

- No progressive recovery after a fair trial of conservative therapy for one to two months,

- Development neurological complication during conservative therapy,

- Neurological status became worse while the patient was undergoing conservative treatment,

- Recurrence of neurological complication,

- Occurrence of prevertebral cervical abscess causing neurological signs and difficulty in deglutition and respiration,

- Advanced neurological involvement such as marked sensory and sphincter disturbances, flaccid paralysis or sever flexor spasms.

ATT was stopped at the end of 6 months as per DOTS schedule and MRI was done at the end of therapy. Following MRI details were noted:
- Cord compression.

- Intramedullary lesion.

- Signal intensity changes in vertebral body and disc space.

- Preservation of disc space.

- Paravertebral, epidural and intraosseous abscess.

\section{RESULTS}

In this study involving 44 patients, 10 were males and 34 were females (Figure 1). In the age group of 10 to 20 years there were 16 patients, in the age group of 21 to 40 years there were 24 patients and in the age group of 41 to 60 years there were 4 patients (Figure 2).

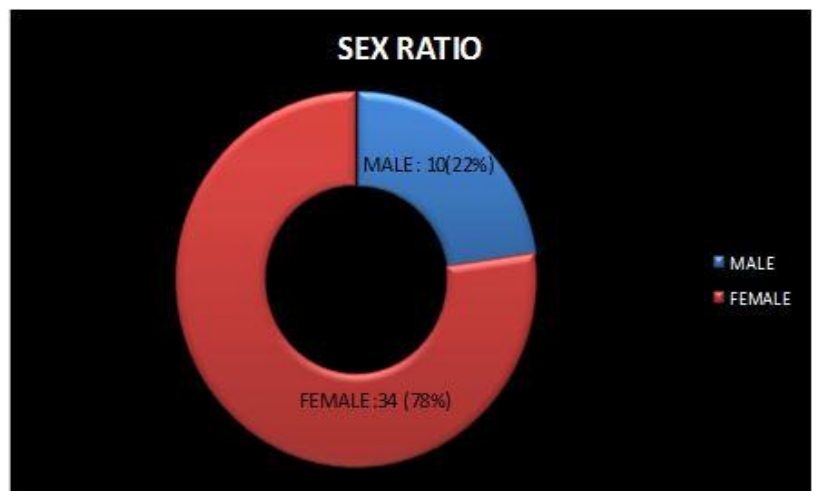

Figure 1: Distribution of sex ratio among patients.

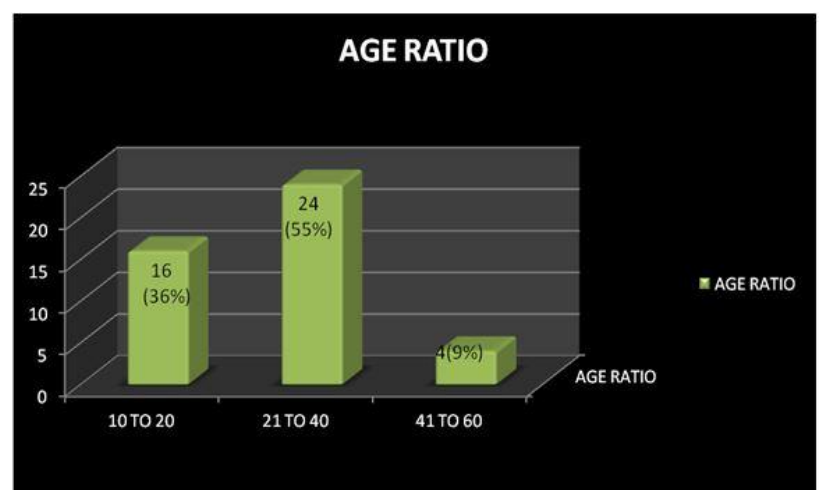

Figure 2: Distribution of age among patients.

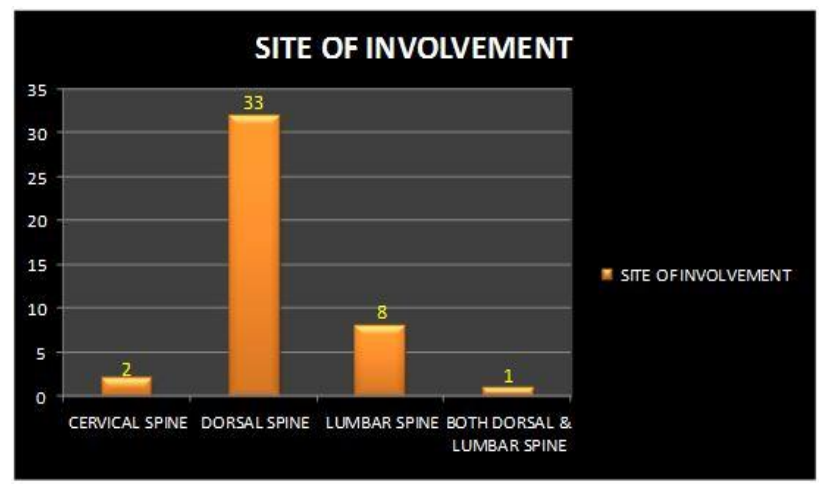

Figure 3: Vertebral level of affection among patients. 


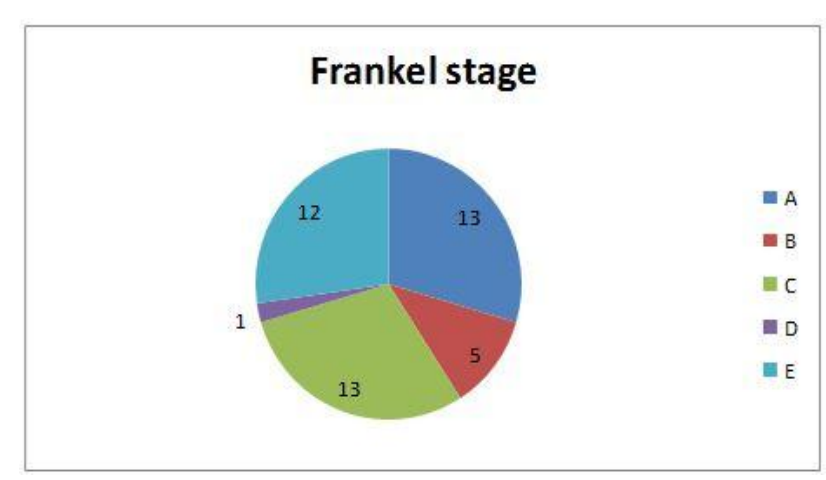

Figure 4: Distribution of patients as per Frankel stage.

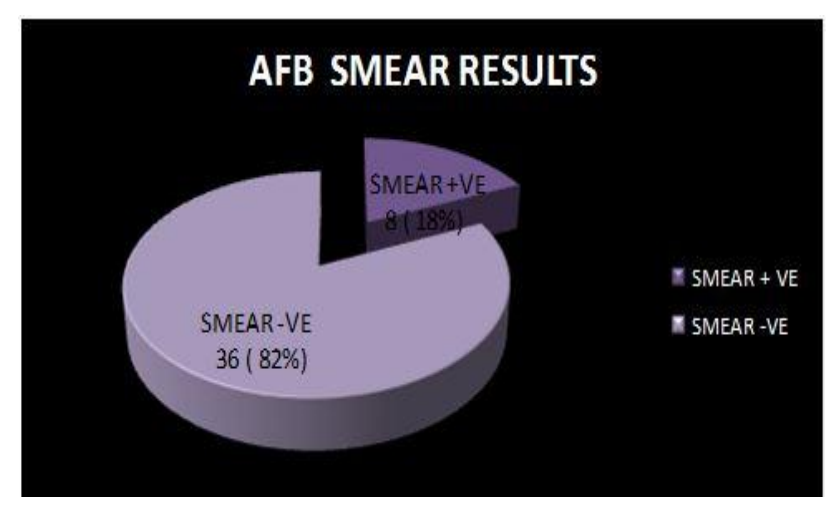

Figure 5: Acid fast bacilli smear results of patients.

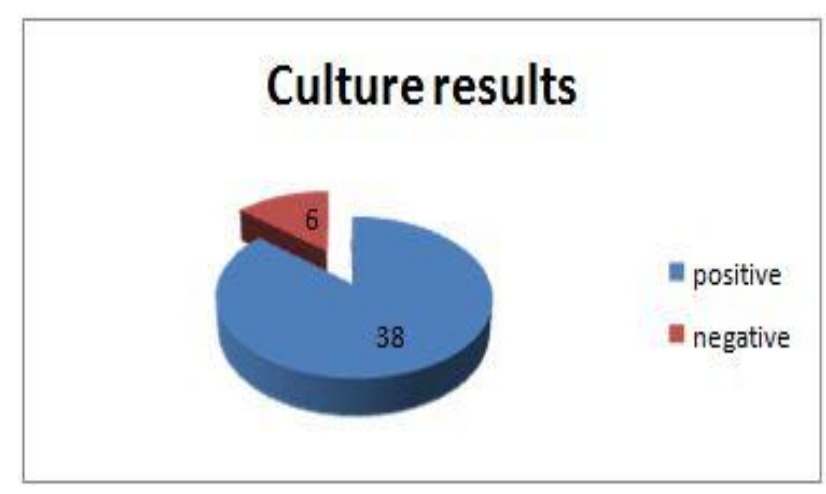

Figure 6: Culture results of patients.

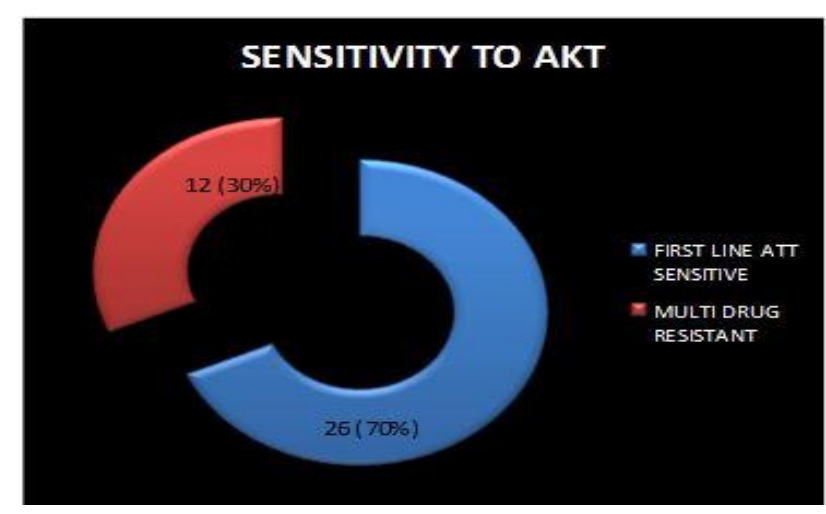

Figure 7: Distribution of sensitivity among patients.
Out of 44 patients, 33 had lesions in the dorsal spine, 8 patients in the lumbar region, 2 in the cervical spine and 1 patient had both dorsal and lumbar spine involvement (skip lesions) (Figure 3). 13 patients in the study presented with complete paraplegia i.e. Frankel grade A. 5 patients presented with Frankel grade B. 13 patients presented with Frankel grade C, 1 patient presented with Frankel grade D and 12 patients presented with Frankel grade E (Figure 4).

Out of 44 patients, $8(18 \%)$ were smear positive, 36 $(82 \%)$ were smear negative for AFB. (Figure 5) 33 patients were culture positive at the end of 3 weeks and 5 patients were culture positive at 6 weeks (Figure 6). Out of 38 culture positive patients, $26(70 \%)$ patients showed culture colonies sensitive to all $1^{\text {st }}$ line ATT drugs. 12 (30\%) patients were resistant to $1^{\text {st }}$ line ATT drugs and hence they had multi drug resistant tuberculosis (MDR) (Figure 7). They were all sensitive to $2^{\text {nd }}$ line ATT drugs.

Out of 44 patients, 36 patients showed classical epitheliod granulomas. For the 6 patients who were culture negative, diagnosis was proved by histopathology which showed caseating granulomas, langhans giant cell and acellular necrotic material.

All the 26 patients who were sensitive to $1^{\text {st }}$ line ATT had satisfactorily results. All the patients were Frankel grade $\mathrm{E}$ at the end of the study but one patient had incomplete resolution on MRI although there was reduction in the amount of prevertebral, paravertebral and epidural abscess compared to the pre- ATT MRI.

One patient, a 50 year old female, was treated for dorsal spine tuberculosis with $1^{\text {st }}$ line ATT and transthoracic decompression. The patients developed hemi paresis at 2 months postoperative despite regular ATT. Culture and sensitivity reported resistance to $1^{\text {st }}$ line ATT. MRI brain done later was suggestive of tuberculoma of the brain. The patient was started on $2^{\text {nd }}$ line ATT and tapering dose of steroids. The neurological status improved.

Of the 12 patients with MDR tuberculosis who were started on $2^{\text {nd }}$ line ATT, eight patients recovered neurologically to Frankel grade E. The remaining four patients did not show any neurological improvement and were still on ATT.

\section{DISCUSSION}

Skeletal tuberculosis mostly occurs during first three decades of life; however no age is immune to it. In affluent societies the disease is being reported essentially in the elderly. In developing countries, diagnosis of tuberculous spondylitis can be reliably made on clinical and radiological examination. However, such a situation is becoming increasingly difficult in affluent countries where tuberculosis is reduced to the status of a rare disease and where the present generation of doctors is unfamiliar with skeletal manifestation of the disease. ${ }^{4}$ In 
such a situation a positive proof of the disease must be obtained employing semi invasive or invasive investigations. In affluent societies corticosteroids, alcoholism, prolonged illness, diabetic state, anti-cancer chemotherapy, immunosuppressive drug therapy in organ transplants and old age are the probable predisposing factors.

While the human immunodefficiency virus (HIV) pandemic has led to a resurgence of tuberculosis, it has had little impact on the epidemiology of spinal tuberculosis. ${ }^{5}$ In a large French study, none of 82 cases of spinal tuberculosis were HIV infected. In another large longitudinal studies among HIV infected patients, few cases of spinal tuberculosis were reported, even after long term follow-up. ${ }^{5,6}$ A study conducted in a New York City hospital between 1985-1995 described 26 patients with spinal tuberculosis. Twenty-seven per cent of them were HIV positive. In our study 1 patient was positive for HIV.

In spinal TB, onset of symptoms is usually insidious and disease progression slow. The usual presentation of patients in our study consisted of pain overlying the affected vertebrae, low-grade fevers, chills, weight loss and nonspecific constitutional symptoms of varying duration. All patients had raised ESR (between 70 to 120) before starting ATT. ESR of all patients who were sensitive to $1^{\text {st }}$ line ATT reduced back to normal (15-30) at the end of ATT. Paraplegia can be the first sign of spinal disease. 13 patients in the study presented with complete paraplegia (Frankel grade A). 5 patients presented with complete loss of power and had only sensory function intact (Frankel grade B). 13 patients had weakness in both lower limbs on presentation (Frankel grade C). 1 patient had fair to good motor below the affected level (Frankel grade D). 12 patients had normal motor and sensory function (Frankel grade E). Varying degrees of weakness, nerve-root compression and sensory involvement can occur. Years after resolution of the initial infection, stretching of spinal cord over bony deformity can lead to paraplegia. Duration of symptoms prior to diagnosis in our patients was from 2 weeks to 1 year. Historically, this interval was at least 12 months on an average, decreasing to between 3 and 6 months in the recent era. ${ }^{7}$

In a series of patients from the southern United States, identified between 1952 and 1972, the average age of patients with spinal TB was 51 years. ${ }^{8}$ The lower thoracic and thoracolumbar spine were the most common areas involved, comprising $48 \%$ to $67 \%$ of lesions. ${ }^{9}$ In our series of 44 patients, all of them were from a metropolitan city. The average age of the patients with spinal tuberculosis was 23.72 years. The youngest patient in our study was 11 years old and oldest was 50 years old. 24 patients were between 20 to 40 years of age. Nowadays, spinal tuberculosis is a disease of children in developing nations and the elderly in the United States and Europe. 41 patients in the study were having lesions in the dorsal and lumbar region. 1 patient had skip lesions. This patient had lesions in the dorsal and the lumbar vertebra with normal vertebrae in between. Isolated cervical involvement is less common. 2 patients in the study had isolated cervical tuberculous spondylitis.

Owing to the distribution of the vertebral arterial supply, involvement of adjacent vertebrae is common. When large vertebral segments or multiple segments with intervening normal zones are present, the paravertebral venous plexus of Batson is implicated. Bony changes are seen two to five months after infection. Usually, the subchondral regions of the vertebral body are affected. When the anterior and lateral portions of the vertebral body are affected, vertebral collapse occurs resulting in kyphosis and gibbus deformity. Involvement of the posterior vertebral body results in cavitation and an extradural mass. Kyphosis occurs in the thoracic spine while the lumbar spine telescopes on itself as it collapses. The disc space is eventually destroyed, but at a slower rate than pyogenic infection.

Tuberculosis can spread from a spinal focus by direct extension through the disc space. Once a paravertebral abscess is formed, the disease can spread via anterior/posterior longitudinal ligaments and extend to the pleural space. From the cervical vertebrae the disease can travel to the posterior triangle of the neck or retropharnyx. The abscess can also track along fascial planes to create a psoas abscess or can extend posteriorly to form an extradural abscess.

Different investigations useful in the diagnosis of tuberculous spondylitis are roentgenogram, blood investigations, Mantoux test, biopsy, guinea pig inoculation, smear and culture, isotope scintigraphy, serological investigations, modern imaging techniques like CT scan and MRI. ${ }^{10}$ The best noninvasive diagnostic modality for spinal tuberculosis is MRI. ${ }^{8,11}$ MRI is more sensitive than radiography and more specific than CT in the diagnosis of spinal tuberculosis. The anatomical pattern revealed by MRI, particularly the soft tissue and disc involvement, yields greater specificity. MRI can also provide the diagnosis of tuberculosis of the spine 4-6 months earlier than conventional methods, offering the benefits of earlier detection and treatment. ${ }^{8}$ MRI allows for the rapid determination of the mechanism for neurologic compression and can distinguish between bone and soft tissue lesion (tuberculoma). Tuberculosis spondylitis typically manifests with juxta-discal body involvement and collapse with paraspinal and epidural pus. The intervertebral disc may have normal height and normal signal on MRI, reflecting the resistance of the disc to tuberculous infection. Gadolinium-contrasted scans may help distinguish abscesses from granulation tissue, with the abscess showing only rim enhancement, whereas the whole mass enhances with granulation tissue. In endemic areas, there may be atypical cases of up to $30 \%$ with a variety of other MRI manifestations. This leads to most lesions with epidural collections being considered as tuberculotis if the patient has a raised ESR 
and is clinically not septicaemic. Reliance on new imaging techniques alone is insufficient.

As radiological appearances are commonly nondiagnostic and imaging studies are not fully reliable for differentiating spinal tuberculosis from other infections or neoplasm, bacteriologic and/or histological confirmation must be obtained. ${ }^{12,13}$ Microbiological diagnosis is virtually impossible without aspiration of pus or some form of tissue sampling. In our study 28 patients were subjected to surgery and their samples (pus, granulation tissue) were collected intra-operatively. Fine needle aspiration biopsy as a diagnostic tool is accurate, safe, and cost effective because the procedure does not require hospitalization. Fine needle aspiration biopsy done under CT-guidance was successful in diagnosing spinal tuberculosis in 16 patients.

After AFB smear, culture and sensitivity report, if the patient turned out to be MDR then patients are started on daily regimen based on the sensitivity to drugs. Of the 44 patients 12 patients turned out to be MDR, they were started on $2^{\text {nd }}$ line ATT drugs.

It is important to remember that tuberculosis can mimic any disease, and any pathology can mimic tuberculosis on clinical features and on imaging modalities. Highest proof of tuberculous disease would be obtained if the diseased material is submitted for direct smear, histology, culture and guinea pig inoculation simultaneously. ${ }^{4,15}$ Stroebel et al reported that enzyme linked immunoabsorbent assay (ELISA) for antibody to mycobacterial antigen-6, demonstrated at a cut off of $1: 32$, sensitivity of $94 \%$ and specificity of $100 \%$ in serologic diagnosis of bone and joint tuberculosis. ${ }^{16}$ Consistency of these observations have not gained acceptance for routine use. Serological investigations are useful in differential diagnosis of brucellosis, typhoid and syphilitic infection.

Whenever there is doubt, it is mandatory to prove the diagnosis by obtaining the disease tissue by core biopsy, needle biopsy or open biopsy and send it for histopathological evaluation which would reveal the typical tubercle. ${ }^{12}$ Culture and sensitivity with histopathological examinations gives the choice of antibiotic and also distinguishes between infective lesions and malignant disease.

Antituberculous chemotherapy is very effective in a compliant patient. The failure of treatment and emergence of drug resistance is due to non-compliance which can only be overcome by establishing a human bond between the patient and the provider through DOTS. In the developing world, evidence from uncontrolled studies shows that the introduction of DOTS has increased completion of therapy and cure rates from $25-50 \%$ (with unsupervised treatment) to $80-90 \%$, with relapse rates of less than $5 \%$. The daily regimen has shown $7 \%$ of hepatotoxicity and about $46 \%$ of severe arthralgias. The incidence of these side effects with
DOTS is less than $2.5 \%$ and $7.3 \%$ respectfully. Intermittent therapy is as effective as daily therapy. ${ }^{3}$ In our study one patient had drugs associated rashes but it subsided on topical steroids.

\section{CONCLUSION}

Tuberculosis still remains a major cause of morbidity and mortality in developing countries. India alone has got one-fifth of the total world population of tuberculous patients. Vertebral tuberculosis is the most common form of skeletal tuberculosis and it contributes about 50 percent of all cases of skeletal tuberculosis. Most important cause of treatment failure and emergence of drug resistance in the treatment of tuberculosis is noncompliance. Compliance can be improved by direct observation of drug intake, intermittent therapy, and short-course treatment known as Direct Observed Treatment Short course (DOTS). By short course chemotheraphy we can avoid side effects of ATT drugs.

We recommend before starting any patient on ATT, it is mandatory to do biopsy, culture, sensitivity and histopathology. Recently there is an increasing tendency in multidrug resistance cases and total drug resistant cases. By doing culture and sensitivity of the specimen we can find out the number of MDR cases and initiate proper drug regimen to avoid further complication and mortality.

\section{Funding: No funding sources} Conflict of interest: None declared

Ethical approval: The study was approved by the institutional ethics committee

\section{REFERENCES}

1. Tuli SM. Epidemiology and prevalence. In: Tuli SM, eds. Tuberculosis of the skeletal system: (Bones, Joints, Spine and Bursal Sheaths), 4rd ed. New Delhi, India: Jaypee Brothers; 2010: 3-8.

2. Tuli SM. Result of treatment of spinal tuberculosis by the "middle path" regime. J Bone Joint Surg. 1975;56:13-23.

3. Valsalan R, Purushothaman R, Raveendran MK, Zacharia B, Surendran S. Efficacy of directly observed treatment short-course intermittent therapy. Indian J Orthop. 2012;46:138-4.

4. Janssens JP, Haller R. Spinal tuberculosis in a developed country. A review of 26 cases with special emphasis on abscesses and neurologic complications. Clin Orthop. 1990;257:67-75.

5. Vassilopoulos D, Chalasani P, Jurado RL, Workowski K, Agudelo CA. Musculoskeletal infections in patients with human immunodeficiency virus infection. Medicine. 1997;76(4):284-94.

6. Munoz FS, Cardenal A, Balsa A. Rheumatic manifestations in 556 patients with human immunodeficiency virus infection. Semin Arthritis Rheum. 1991;21:30-9. 
7. Lifeso RM, Weaver P, Harder EH. Tuberculous spondylitis in adults. J Bone Joint Surg Am 1985;67:1405-13.

8. Desai SS. Early diagnosis of spinal tuberculosis by MRI. J Bone Joint Surg Br. 1994;76:863-9.

9. Moon MS, Woo YK, Lee KS, Ha KY, Kim SS, Sun $\mathrm{DH}$. Posterior instrumentation and anterior interbody fusion for tuberculous kyphosis of dorsal and lumbar spines. Spine. 1995;20(17):1910-6.

10. Cotton A, Flipo RM, Drouot MH. Spinal tuberculosis: Study of the radiological aspects of 82 cases. J Radiol. 1996;77:419-26.

11. Ahmadi J, Bajaj A, Destian S. Spinal tuberculosis: atypical observations at MR imaging. Radiology. 1993;189:489-93.

12. Boachie-Adjei O, Squillante RG. Tuberculosis of the spine. Orthop Clin North Am. 1996;27:95-103.

13. Su SH1, Tsai WC, Lin CY, Lin WR, Chen TC, Lu $\mathrm{PL}$, et al. Clinical features and outcomes of spinal tuberculosis in Southern Taiwan. J Microbiol Immunol. Infect. 2010;43(4):291-300.
14. Lakhanpal VP, Tuli SM, Singh H, Sen PC. The value of histology, culture and guinea pig inoculation examination in osteo-articular tuberculosis. Acta Orthopaedica Scandinavica. 1974;45:36-42.

15. Hald J. The Value of histological and bacteriological examination in tuberculosis of bones and joints. Acta Orthopaedica Scandinavica. 1965;35:1-97.

16. Stroebel AB, Daniel TM, Lau JHK, Leong JCY, Richardson H. Serologic diagnosis of bone and joint tuberculosis by an enzyme-linked immunosorbent assay. J Infect Dis. 1982;146(2):280-3.

Cite this article as: Jagiasi JD, Patel MR. Results of management of spinal tuberculosis according to middle path regime and short course chemotheraphy. Int J Res Orthop 2017;3:966-72. 